\title{
Chemoenzymatic synthesis of a phosphorylated glycoprotein**
}

\author{
Pragya Priyanka, Thomas B. Parsons, Antonia Miller, Frances M. Platt, and Antony J. Fairbanks*
}

\begin{abstract}
The majority of lysosomal enzymes are targeted to the lysosome by post-translational tagging with $\mathrm{N}$-glycans terminated in mannose-6-phosphate (M6P) residues. Some current enzyme replacement therapies (ERTs) for lysosomal storage disorders are limited in their efficacy by the extent to which recombinant enzymes bear the M6P terminated glycans required for effective trafficking. A combination of chemical synthesis and endo- $\beta$ - $N$-acetylglucosaminidase (ENGase) catalysis allows the convergent synthesis of glycosyl amino acids bearing M6P residues. The approach can be extended to the remodelling of proteins, as exemplified by $R$ Nase. The powerful synergy of chemical synthesis and ENGase mediated biocatalysis allows the first synthesis of a glycoprotein bearing M6P terminated N-glycans in which the glycans are attached to the peptide backbone by entirely natural linkages.
\end{abstract}

Phosphorylation of the terminal mannose residues of $\mathrm{N}$ glycans is a post-translational protein modification that occurs via a two-step process involving the sequential action of two enzymes. [1] The primary purpose of the installation of mannose-6-phosphate (M6P) at the non-reducing termini of high mannose oligosaccharides is to facilitate the trafficking of proteins carrying such 'tagged' glycans to the lysosome via interaction with the mannose-6-phosphate receptors (M6PRs). ${ }^{[2]}$

[*] P. Priyanka, A. J. Fairbanks Department of Chemistry University of Canterbury Private Bag 4800, Christchurch 8140, New Zealand. Fax: +64 33642110

E-mail: antony.fairbanks@canterbury.ac.nz

T.B. Parsons

Department of Chemistry, Chemistry Research Laboratory, University of Oxford, Mansfield Road, Oxford, OX1 3TA, UK A. Miller

Callaghan Innovation, School of Biological Sciences, University of Canterbury, Private Bag 4800, Christchurch 8140, New Zealand

F. M. Platt

Department of Pharmacology, University of Oxford, Mansfield Road, Oxford, OX1 3QT, UK

[**] The authors thank Dr Marie Squire for technical assistance, and the EPSRC (DTA studentship to TBP) for funding. We also thank Professors David Vocadlo (Simon Fraser University, British Columbia, Canada) and Alisdair Boraston (University of Victoria, British Columbia, Canada) for kindly providing the Endo D enzyme.

Supporting information for this article is available on the WWW under http://www.angewandte.org or from the author.((Please delete if not appropriate))
Targeting of enzymes to the lysosome via the M6PRs is of critical interest for the development and enhancement of enzyme replacement therapies (ERTs) for the treatment of lysosomal storage disorders (LSDs). For example, a variety of avenues of investigation have been pursued to try and enhance the delivery of recombinant human acid $\alpha$-glucosidase (rhGAA, commercially marketed as Myozyme) to affected muscles in order to increase the efficacy of ERT for the treatment of Pompe disease by targeting via the M6PR. Approaches have included the chemical conjugation of naturally derived or synthetic oligosaccharides containing M6P residues, ${ }^{[3][4]}$ as well the enzymatic addition of M6P residues. ${ }^{[5]}$ The total synthesis of a variety of $\mathrm{N}$-glycan structures containing M6P residues has also been reported, and these have been attached to a protein via a non-native linker. ${ }^{[6]}$

Amongst various synthetic approaches developed to access glycoproteins, ${ }^{[7]}$ the use of endo- $\beta-\mathrm{N}$-acetylglucosaminidases (ENGases) has allowed the synthesis of a variety of homogenous glycopeptides, proteins and antibody fragments containing N-glycan structures. ${ }^{[8]}$ Recent advances such as the use of oxazolines as activated donors, ${ }^{[9]}$ methodology that allows the synthesis of these oxazolines in water from the corresponding reducing sugars, ${ }^{[10]}$ and the production of efficient glycosynthase mutant enzymes, ${ }^{[11]}$ have all greatly increased the utility of this approach. However whether this strategy can be applied to access glycoconjugates that contain phosphorylated saccharides has not been investigated. As part of on-going exploration into the utility of ENGases, a program was embarked upon in which the synthesis of $\mathrm{N}$-glycan structures containing M6P residues was undertaken, and the ability of ENGases to catalyse their transfer to peptides and proteins was investigated.

Studies into interaction of glycans with the M6PRs have shown that multivalent presentation of terminal M6P residues significantly increases receptor binding. [12] An N-glycan pentasaccharide structure in which the two terminal mannose residues contained phosphate at the 6-position was identified as a preliminary synthetic target; with this material it would be possible to assay whether ENGases could process glycans containing M6P residues before undertaking more complicated and arduous syntheses of the fully extended structures required for optimal binding to the M6PRs. The synthetic strategy adopted was based upon sequences previously used to access N-glycan oxazolines. ${ }^{[9 j]}$ However, in this case the installation of a phosphate group at position- 6 of mannose residues at the non-reducing terminus required a selective protection strategy. Thus OH-6 of the known tetrol $\mathbf{1}^{[13]}$ was selectively protected by reaction with TIPS chloride to give triol 2; this was then benzylated to give thioglycoside $\mathbf{3}$, which was to serve as a 


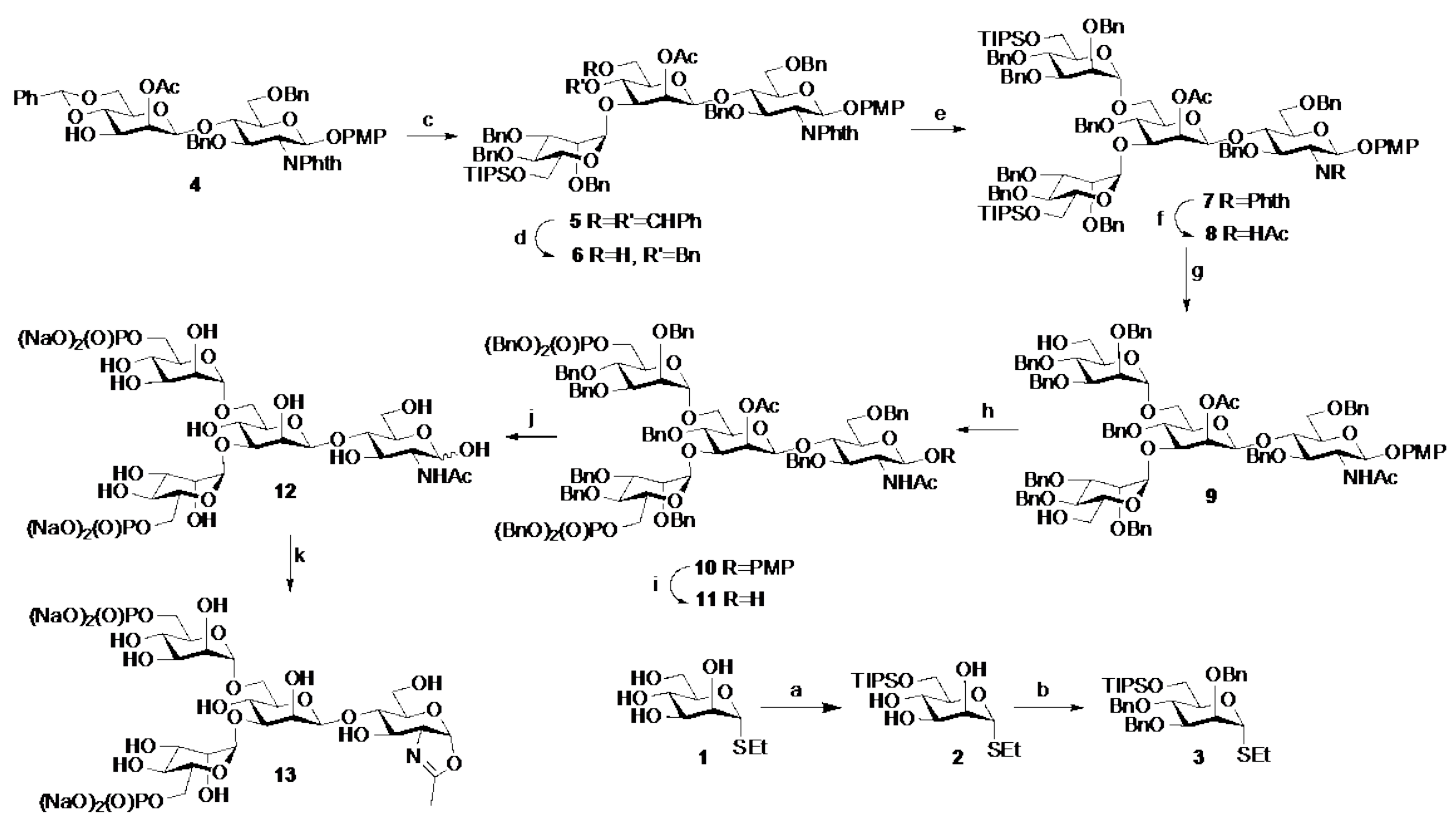

Scheme 1. Synthesis of oxazoline 13 (a) TIPSCl, imidazole, THF, $0{ }^{\circ} \mathrm{C}$ to rt, $24 \mathrm{~h}, 89 \%$; (b) $\mathrm{NaH}, \mathrm{BnBr}, \mathrm{THF}, 60{ }^{\circ} \mathrm{C}, 24 \mathrm{~h}, 78 \%$; (c) 3, MeOTf, $3 \AA$ mol. sieves, DCM, t-TBP, $0{ }^{\circ} \mathrm{C}$ to rt, 18 h, 91\%; (d) $\mathrm{Et}_{3} \mathrm{SiH}, \mathrm{PhBCl}_{2}, 3 \AA$ mol. sieves, DCM, $-78{ }^{\circ} \mathrm{C}, 50 \mathrm{~min}$, 94\%; (e) 3, MeOTf, $3 \AA ̊$ mol. sieves, DCM, $t$-TBP, $0{ }^{\circ} \mathrm{C}$ to rt, 18 h, 79\%; (f) (i) $\mathrm{NH}_{2} \mathrm{CH}_{2} \mathrm{CH}_{2} \mathrm{NH}_{2}, \mathrm{MeOH}, \mathrm{reflux}, 16$ h; (ii) Ac $2 \mathrm{O}$, py, rt, 24 h, 91\% over 2 steps; (g) BF $3 . \mathrm{OEt}_{2}, \mathrm{DCM}, 0{ }^{\circ} \mathrm{C}, 1 \mathrm{~h}, 76 \%$; (h) (BnO) ${ }_{2} \mathrm{PNiPr}_{2}$, tetrazole, DCM, rt, $18 \mathrm{~h}$, then add MCPBA, $-78{ }^{\circ} \mathrm{C}$ to rt, 2 h, 58\% over two steps; (i) CAN, $\mathrm{MeCN}_{2} \mathrm{H}_{2} \mathrm{O}$, rt, 1 h, 78\%; (j) Na, $\mathrm{NH}_{3}(\mathrm{l}),-33{ }^{\circ} \mathrm{C}, 1$ h, $98 \%$; (k) DMC, Et $3 \mathrm{~N}, \mathrm{H}_{2} \mathrm{O}, \mathrm{rt}$, $95 \%$.

common donor for introduction of both the 3- and 6-branches of the core N-glycan oligosaccharide. The acceptor disaccharide 4 was accessed as previously described, ${ }^{[9 \mathrm{k}]}$ and glycosylation of $\mathbf{4}$ with $\mathbf{3}$ produced trisaccharide $\mathbf{5}$. Regioselective reductive cleavage of the 4,6-benzylidene, gave the primary alcohol 6, and was followed by a second glycosylation with donor $\mathbf{3}$ to yield tetrasaccharide $\mathbf{7}$ (Scheme 1). Conversion of the $N$-phthalimide to the $N$-acetamide gave $\mathbf{8}$, and was followed by removal of the TIPS groups to produce diol 9. Phosphitylation of $\mathbf{9}$ with dibenzyl diisopropylphosphoramidate and immediate oxidation with MCBPA gave the bis phosphate ester 10. Oxidative cleavage of the anomeric PMP protecting group then gave hemiacetal 11, which was globally de-protected by Birch reduction to yield free sugar 12 in very high yield. Finally, 12 was converted into the corresponding oxazoline $\mathbf{1 3}$ following the procedure of Shoda $^{[10]}$ using 2-chloro-1,3-dimethylimidazolinium chloride (DMC) in water in $95 \%$ yield.

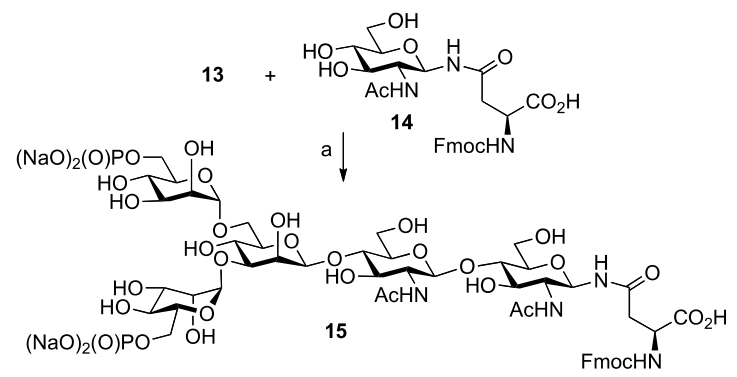

Scheme 2. ENGase catalysed glycosylation of 14 (a) ENGase, phosphate buffer $\mathrm{pH} 6.5, \mathrm{H}_{2} \mathrm{O}, 37^{\circ} \mathrm{C}, 2 \mathrm{~h}$.
Oxazoline 13 was investigated as a substrate for several of the family GH85 ENGase enzymes, using the glycosyl amino acid 14 as acceptor (Scheme 2). Wild-type (WT) Endo $A^{[14]}$ was able to catalyse glycosylation of $\mathbf{1 4}$, and with three equivalents of donor 13, product 15 was formed in $40 \%$ yield after 2 hours. Increasing the number of equivalents of $\mathbf{1 3}$ from three to six correspondingly increased the yield of $\mathbf{1 5}$ to a very respectable $73 \%$. A time course study of the reaction revealed that although 15 was a hydrolytic substrate for WT Endo A, hydrolysis occurred only slowly, allowing effective kinetic control and isolation in high yield (Figure 1).

Other family GH85 enzymes assayed were found to be less effective. Interestingly WT Endo $\mathrm{M}^{[15]}$ was unable to catalyse the formation of 15, a result which was unexpected as Endo M has a broader hydrolytic capability than Endo A, being able to hydrolyse complex biantennary $\mathrm{N}$-glycans as well as high mannose structures. The fact that complex $\mathrm{N}$-glycans are terminated by negatively charged sialic acid residues had led us to speculate that perhaps Endo M may be more tolerant of high mannose glycans terminated in negatively charged phosphates, but, with the tetrasaccharide oxazoline 13, this was not the case. Similarly the commercially available N175Q glycosynthase mutant of Endo $\mathrm{M}^{[11 \mathrm{~d}]}$ was also unable to effect glycosylation of $\mathbf{1 4}$ with oxazoline 13. On the other hand, Endo $\mathrm{D}$, which has considerably more specific structural requirements with respect to the $\mathrm{N}$-glycans it will hydrolyse, was able to catalyse the production of $\mathbf{1 5}$, albeit in a very low yield ( 3\%). 


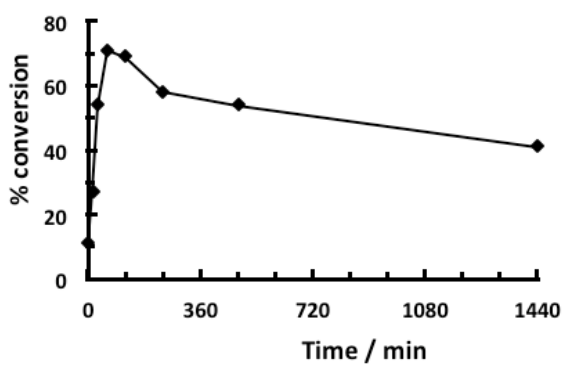

Figure 1. Time course study for the conversion of $\mathbf{1 4}$ into 15 with WT Endo A.

Attention turned to application of WT Endo A catalysed glycosylation using $\mathbf{1 3}$ to the production of a phosphorylated glycoprotein. RNase B was chosen as the model protein substrate. Commercially available RNase B, which is a mixture of high mannose glycoforms, was trimmed back to a single GlcNAc residue at the sole glycosylation site by treatment with Endo $\mathrm{H}$ as previously described, ${ }^{[11 \mathrm{~b}]}$ to give dRNase B 16. Treatment of 16 with 3 equivalents of oxazoline 13 and WT Endo A gave the phosphorylated glycoprotein (M6P) ${ }_{2}$ RNase 17 (Scheme 3) as demonstrated by SDS PAGE and HRMS (Supporting information Figures S2 and S3).

The successful glycosylation of dRNase B $\mathbf{1 6}$ with oxazoline 13 represents the first report of the synthesis of an N-linked glycoprotein that contains M6P-terminated glycans in which the oligosaccharides are linked to the peptide by native

Keywords: carbohydrates · glycoproteins · ENGase · mannose-6phosphate $\cdot \mathrm{N}$-glycans

[1] M. L. Reitman, S. Kornfeld, J. Biol. Chem. 1981, 256, 11977-80.

[2] There are two M6PRs: the cation dependent mannose 6-phosphate receptor (CD-M6PR) and the cation independent mannose 6phosphate receptor (CI-M6PR).

[3] Y. Zhu, J.-L. Jiang, N. K. Gumlaw, J. Zhang, S. D. Bercury, R. J. Ziegler, K. Lee, M. Kudo, W. M. Canfield, T. Edmunds, C. Jiang, R. J. Mattaliano, S. H. Cheng, Mol. Ther. 2009, 17, 954-963.

[4] a) Y. Zhu, X. Li, J. Kyazike, Q. Zhou, B. L Thurberg, N. Raben, R. J. Mattaliano, S. H. Cheng, J. Biol. Chem. 2004, 279, 50336-41; b) Y.

Zhu, X. Li, A. McVie-Wylie, C. Jiang, B. L. Thurberg, N. Raben, R. J. Mattaliano, S. H .Cheng, Biochem. J. 2005, 389, 619-28.

[5] A. J. McVie-Wylie, K. L. Lee, H. Qiu, X. Jin, H. Do, R. Gotschall, B. L. Thurberg, C. Rogers, N. Raben, M. O'Callaghan, W. Canfield, L. Andrews, J. M. McPherson, R. J. Mattaliano, Mol. Genet. Metab. 2008, 94, 448-55.

[6] a) Y. Liu, Y. M. Chan, J. Wu, C. Chen, A. Benesi, J. Hu, Y. Wang, G. Chen, Chembiochem 2011, 12, 685-690; b) Y. Liu, J. Marshall, Q. Li, N. Edwards, G. Chen, Bioorg. Med. Chem. Lett. 2013, 23, 2328-31.

[7] See for example: a) C. Unverzagt, Y. Kajihara, Chem. Soc. Rev. 2013, 42, 4408-4420; b) S. Dedola, M. Izumi, Y. Makimura, A. Seko, A. Kanamori, M. Sakono, Y. Ito, Y. Kajihara, Angew. Chem. Int. Ed. 2014, 53, 2883-2887; c) P. Wang, S. Dong, J. -H. Shieh, E. Peguero, R. Hendrickson, M. A. S. Moore, S. J. Danishefsky, Science 2013 342, 1357-1360; d) A. Reif, S. Siebenhaar, A. Tröster, M. Schmälzlein, C. Lechner, P. Velisetty, K. Gottwald, C. Pöhner, I. Boos, V. Schubert, S. Rose-John, C. Unverzagt, Angew. Chem. Int. Ed. 2014, 53, 1212512131; e) C. Piontek, D. V. Silva, C. Heinlein, C. Pöhner, S. Mezzato, linkages. Such a strategy should allow the production of recombinant lysosomal hydrolases decorated with M6P terminated glycans, and so may augment the efficiency of trafficking of administered enzymes to the lysosome via the M6PR; a development that could have significance for improving the efficacy of the treatment of LSDs by ERT. The synthesis of full-length $\mathrm{N}$-glycan structures bearing M6P residues of optimal structure for lysosomal targeting via the M6PR, and their biocatalytic attachment to glycopeptides and glycoproteins is currently in progress, and the results will be reported in due course.

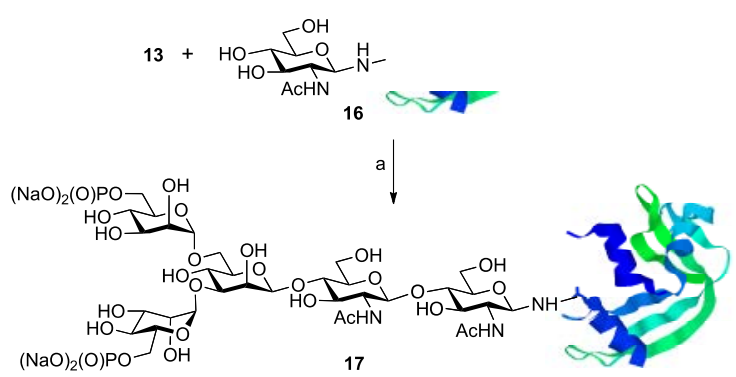

Scheme 3 Enzymatic synthesis of remodelled RNase. a) WT-Endo A, phosphate buffer $\mathrm{pH} 6.5, \mathrm{H}_{2} \mathrm{O}, 37^{\circ} \mathrm{C}, 2 \mathrm{~h}$

P. Ring, A. Martin, F. X. Schmid, C Unverzagt, Angew. Chem. Int. Ed. 2009, 48, 1941-1945.

[8] a) L.-X. Wang, Carbohydr. Res. 2008, 343, 1509-1522; b) A. J. Fairbanks, Comptes Rendus Chimie 2011, 14, 44-58; c) A. J. Fairbanks, Pure Appl. Chem. 2013, 85, 1847-1863.

[9] a) M. Fujita, S. -i. Shoda, K. Haneda, T. Inazu, K. Takegawa, K. Yamamoto, Biochim. Biophys. Acta 2001, 1528, 9-14; b) B. Li, Y. Zeng, S. Hauser, H. Song, L. -X. Wang, J. Am. Chem. Soc. 2005, 127, 9692-3; c) L.-X. Wang, H. J. Song, S. W. Liu, H. Lu, S. B. Jiang, J. H. Ni, H. G. Li, ChemBioChem 2005, 6, 1068-1074; d) Y. Zeng, J. S. Wang, B. Li, S. Hauser, H. G. Li, L.-X. Wang, Chem. Eur. J. 2006, 12, 3355-3364; e) B. Li, H. Song, S. Hauser, L.-X. Wang, Org. Lett. 2006, 8, 3081-3084. f) W. Huang, H. Ochiai, X. Zhang, L.-X. Wang, Carbohydr. Res. 2008, 343, 2903-2913; g) H. Ochiai, W. Huang, L.X. Wang, Carbohydr. Res., 2009, 344, 592-598; h) T. W. D. F. Rising, T. D. W. Claridge, N. Davies, D. P. Gamblin, J. W. B. Moir, A. J. Fairbanks, Carbohydr. Res. 2006, 341, 1574-1596; i) T. W. D. F. Rising, T. D. W. Claridge, J. W. B. Moir, A. J. Fairbanks, ChemBioChem 2006, 7, 1177-1180; j) T. W. D. Rising, C. D.; Heidecke, J. W. B. Moir, Z. Ling, A. J. Fairbanks, Chem. Eur. J. 2008 14, 6444-6464; k) T. B. Parsons, J. W. B. Moir, A. J. Fairbanks, Org. Biomol. Chem. 2009, 7, 3128-3140; l) Y. Tomabechi, G. Krippner, P M. Rendle, M. A. Squire, A. J. Fairbanks Chem. Eur. J. 2013, 19, 15084 - 15088; m) R. Kowalczyk, M. A. Brimble, Y. Tomabechi, A. J. Fairbanks, M. Fletcher, D. L. Hay, Org. Biomol. Chem. 2014, 12, 8142-8151; n) J. D. McIntosh, M. A. Brimble, A. E. S. Brooks, P. R. Dunbar, R. Kowalczyk, Y. Tomabechi, A. J. Fairbanks, Chem. Sci. 2015, 6, 4636-4642.

[10] M. Noguchi, T. Tanaka, H. Gyakushi, A. Kobayashi, S. -i. Shoda, J. Org. Chem. 2009, 74, 2210-2212.

[11] a) M. Umekawa, W. Huang, B. Li, K. Fujita, H. Ashida, L. -X. Wang, K. Yamamoto, J. Biol. Chem. 2008, 283, 4469-4479; b) C. D. Heidecke, Z. Ling, N. C. Bruce, J. W. B. Moir, T. B.; Parsons, A. J. Fairbanks, ChemBioChem, 2008, 9, 2045-2051; c) W. Huang, C. Li, B. Li, M. Umekawa, K. Yamamoto, X. Zhang, L. -X. Wang, J. Am. Chem. Soc. 2009, 131, 2214-2223; d) M. Umekawa, C. Li, T. 
Higashiyama, W. Huang, H. Ashida, K. Yamamoto, L. -X. Wang, J. Biol. Chem. 2010, 285, 511-521.

[12] a) J. J. Distler, J. F. Guo, G. W. Jourdian, O. P. Srivastava, O. Hindsgaul, J. Biol. Chem. 1991, 266, 21687-21692; b) P. Ghosh, N. M. Dahms, S. Kornfeld, Nat. Rev. Mol. Cell Biol. 2003, 4, 202-213.

[13] J. Fried, D. E. Walz, J. Am. Chem. Soc. 1949,71, 140-143.

[14] a) K. Takegawa, M. Nakoshi, S. Iwahara, K. Yamamoto, T. Tochikura, Appl. Environ. Microbiol., 1989, 55, 3107-3112. b) K. Takegawa, M. Tabuchi, S. Yamaguchi, A. Kondo, I. Kato, S. Iwahara, J. Biol. Chem., 1995, 270, 3094-3099. c) J.-Q. Fan, K. Takegawa, S. Iwahara, A. Kondo, I. Kato, C. Abeygunawardana, Y. C. Lee, J. Biol. Chem., 1995, 270, 17723-17729. d) J.-Q. Fan, M. S. Quesenberry, K. Takegawa, S. Iwahara, A. Kondo, I. Kato, Y. C. Lee, J. Biol. Chem., 1995, 270, 17730-177359.

[15] a) K. Yamamoto, S. Kadowaki, J. Watanabe, H. Kumagai, Biochem. Biophys. Res. Commun., 1994, 203, 244-252. b) K. Yamamoto, S.

Kadowaki, M. Fujisaki, H. Kumagai, T. Tochikura, Biosci. Biotech. Biochem., 1994, 58, 72-77. c) K. Haneda, T. Inazu, K. Yamamoto, H.
Kumagai, Y. Nakahara, A. Kobata, Carbohydr. Res., 1996, 292, 61-70. d) K. Yamamoto, K. Fujimori, K. Haneda, M. Mizuno, T. Inazu, H. Kumagai, Carbohydr. Res., 1998, 305, 415-422.

[16] C. M. Eng, N. Guffon, W. R. Wilcox, D. P. Germain, P. Lee, S. Waldek, L. Caplan, G. E. Linthorst, R. J. Desnick, N. Engl. J. Med. 2001, 345, 9 - 16.

[17] S. C Garman, D. N. Garboczi, J. Mol. Biol. 2004, 337, 319-335.

[18] K. Lee, X. Jin, K. Zhang, L. Copertino, L. Andrews, J. BakerMalcolm, L. Geagan, H. Qiu, K. Seiger, D. Barngrover, J. M. McPherson, T. Edmunds, Glycobiology 2003, 13, 305-313. 
Entry for the Table of Contents (Please choose one layout)

Layout 1:

\section{((Catch Phrase))}

((Author(s), Corresponding Author(s) $\left.{ }^{\star}\right)$ )

Page - Page

((Title Text))

((TOC Graphic))

((Text for Table of Contents, max. 450 characters))

Layout 2:

\section{Glycoproteins}

Pragya Priyanka, Thomas B. Parsons, Antonia Miller, Frances M. Platt, and Antony J. Fairbanks* Page - Page

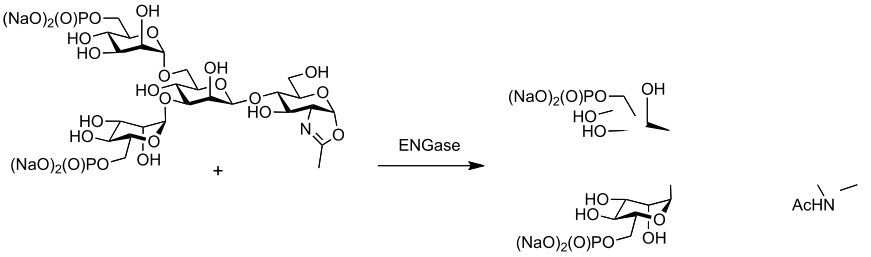

Chemoenzymatic synthesis of a phosphorylated glycoprotein

ENGase-ineering glycoproteins. The combination of chemical synthesis and ENGase-mediated biocatalysis allows the production of a glycoprotein bearing mannose-6-phosphate terminated N-glycans that are linked to the peptide backbone by natural linkages. 


\section{Word references - please ignore}

${ }^{1}$ Reitman M. L.; Kornfeld S. J. Biol. Chem. 1981, 256, 11977-80.

${ }^{2}$ There are two M6PRs: the cation dependent mannose 6-phosphate receptor (CD-M6PR) and the cation independent mannose 6-phosphate receptor (CIM6PR).

${ }^{3}$ Y. Zhu, J.-L. Jiang, N. K. Gumlaw, J. Zhang, S. D. Bercury, R. J. Ziegler, K. Lee, M. Kudo, W. M. Canfield, T. Edmunds, C. Jiang, R. J. Mattaliano, S. H. Cheng Mol. Ther. 2009, 17, 954-963.

${ }^{4}$ a) Yunxiang Zhu, Xuemei Li, Josephine Kyazike, Qun Zhou, Beth L Thurberg, Nina Raben, Robert J Mattaliano, Seng H Cheng J. Biol. Chem. 2004, 279, 50336-41; b) Yunxiang Zhu, Xuemei Li, Alison McVie-Wylie, Canwen Jiang, Beth L Thurberg, Nina Raben, Robert J Mattaliano, Seng H Cheng, Biochem. J. 2005, 389, 619-28.

${ }^{5}$ A. J. McVie-Wylie, K. L. Lee, H. Qiu, X. Jin, H. Do, R. Gotschall, B. L. Thurberg, C. Rogers, N. Raben, M. O'Callaghan, W. Canfield, L. Andrews, J. M. McPherson, R. J. Mattaliano, Mol. Genet. Metab. 2008, 94, 448-55.

${ }^{6}$ a) Y. Liu, Y. M. Chan, J. Wu, C. Chen, A. Benesi, J. Hu, Y. Wang, G. Chen, Chembiochem 2011, 12, 685-690; b) Y. Liu, J. Marshall, Q. Li, N. Edwards, G. Chen, Bioorg. Med. Chem. Lett. 2013, 23, 2328-31.

${ }^{7}$ See for example: a) C. Unverzagt, Y. Kajihara, Chem. Soc. Rev. 2013, 42, 4408-4420; b) S. Dedola, M. Izumi, Y. Makimura, A. Seko, A. Kanamori, M. Sakono, Y. Ito, Y. Kajihara, Angew. Chem. Int. Ed. 2014, 53, 2883-2887; c) P. Wang, S. Dong, J. -H. Shieh, E. Peguero, R. Hendrickson, M. A. S. Moore, S. J. Danishefsky, Science 2013, 342, 1357-1360; d) A. Reif, S. Siebenhaar, A. Tröster, M. Schmälzlein, C. Lechner, P. Velisetty, K. Gottwald, C. Pöhner, I. Boos, V. Schubert, S. Rose-John, C. Unverzagt, Angew. Chem. Int. Ed. 2014, 53, 12125-12131; e) C. Piontek, D. V. Silva, C. Heinlein, C. Pöhner, S. Mezzato, P. Ring, A. Martin, F. X. Schmid, C Unverzagt, Angew. Chem. Int. Ed. 2009, 48, 1941-1945.

${ }^{8}$ a) L.-X. Wang, Carbohydr. Res. 2008, 343, 1509-1522; b) A. J. Fairbanks, Comptes Rendus Chimie 2011, 14, 44-58.

${ }^{9}$ a) M. Fujita, S. -i. Shoda, K. Haneda, T. Inazu, K. Takegawa, K. Yamamoto, Biochim. Biophys. Acta 2001, 1528, 9-14; b) B. Li, Y. Zeng, S. Hauser, H. Song, L. -X. Wang, J. Am. Chem. Soc. 2005, 127, 9692-3; c) L.-X. Wang, H. J. Song, S. W. Liu, H. Lu, S. B. Jiang, J. H. Ni, H. G. Li, ChemBioChem 2005, 6, 1068-1074; d) Y. Zeng, J. S. Wang, B. Li, S. Hauser, H. G. Li, L.-X. Wang, Chem. Eur. J. 2006, 12, 3355-3364; e) B. Li, H. Song, S. Hauser, L.X. Wang, Org. Lett. 2006, 8, 3081-3084. f) W. Huang, H. Ochiai, X. Zhang, L.-X. Wang, Carbohydr. Res. 2008, 343, 2903-2913; g) Y. Wei, C. Li, W. Huang, B. Li, S. Strome, L.-X. Wang, Biochemistry 2008, 47, 10294-10304; h) H. Ochiai, W. Huang, L.-X. Wang, Carbohydr. Res., 2009, 344, 592-598; i) T. W. D. F. Rising, T. D. W. Claridge, N. Davies, D. P. Gamblin, J. W. B. Moir, A. J. Fairbanks, Carbohydr. Res. 2006, 341, 1574-1596; j) T. W. D. F. Rising, T. D. W. Claridge, J. W. B. Moir, A. J. Fairbanks, ChemBioChem 2006, 7, 1177-1180; k) T. W. D. Rising, C. D.; Heidecke, J. W. B. Moir, Z. Ling, A. J. Fairbanks, Chem. Eur. J. 2008, 14, 6444-6464. l) T. B. Parsons, J. W. B. Moir, A. J. Fairbanks, Org. Biomol. Chem. 2009, 7, 3128-3140.

${ }^{10}$ M. Noguchi, T. Tanaka, H. Gyakushi, A. Kobayashi, S. -i. Shoda, J. Org. Chem. 2009, 74, 2210-2212.

11 a) M. Umekawa, W. Huang, B. Li, K. Fujita, H. Ashida, L. -X. Wang, K. Yamamoto, J. Biol. Chem. 2008, 283, 4469-4479; b) C. D. Heidecke, Z. Ling, N. C. Bruce, J. W. B. Moir, T. B.; Parsons, A. J. Fairbanks, ChemBioChem, 2008, 9, 2045-2051; c) W. Huang, C. Li, B. Li, M. Umekawa, K. Yamamoto, X. Zhang, L. -X. Wang, J. Am. Chem. Soc. 2009, 131, 2214-2223; d) M. Umekawa, C. Li, T. Higashiyama, W. Huang, H. Ashida, K. Yamamoto, L. -X. Wang, J. Biol. Chem. 2010, 285, 511-521.

12 a) J. J. Distler, J. F. Guo, G. W. Jourdian, O. P. Srivastava, O. Hindsgaul, J. Biol. Chem. 1991, 266, 21687-21692; b) P. Ghosh, N. M. Dahms, S. Kornfeld, Nat. Rev. Mol. Cell Biol. 2003, 4, 202-213.

${ }^{13}$ Fried, J.; Walz, D. E. J. Am. Chem. Soc. 1949,71, 140-143.

${ }^{14}$ a) K. Takegawa, M. Nakoshi, S. Iwahara, K. Yamamoto and T. Tochikura, Appl. Environ. Microbiol., 1989, 55, 3107-3112. b) K. Takegawa, M. Tabuchi, S. Yamaguchi, A. Kondo, I. Kato and S. Iwahara, J. Biol. Chem., 1995, 270, 3094-3099. c) J.-Q. Fan, K. Takegawa, S. Iwahara, A. Kondo, I. Kato, C. Abeygunawardana and Y. C. Lee, J. Biol. Chem., 1995, 270, 17723-17729. d) J.-Q. Fan, M. S. Quesenberry, K. Takegawa, S. Iwahara, A. Kondo, I. Kato, and Y. C. Lee, J. Biol. Chem., 1995, 270, 17730-177359. e) J.-Q. Fan, L. H. Huynh, B. B. Reinhold, V. N. Reinhold, K. Takegawa, S. Iwahara, A. Kondo, I. Kato and Y. C. Lee, Glycoconjugate J., 1996, 13, 643-652.

${ }^{15}$ a) K. Yamamoto, S. Kadowaki, J. Watanabe and H. Kumagai, Biochem. Biophys. Res. Commun., 1994, 203, 244-252. b) K. Yamamoto, S. Kadowaki, M. Fujisaki, H. Kumagai and T. Tochikura, Biosci. Biotech. Biochem., 1994, 58, 72-77. c) K. Haneda, T. Inazu, K. Yamamoto, H. Kumagai, Y. Nakahara and A. Kobata, Carbohydr. Res., 1996, 292, 61-70. d) K. Yamamoto, K. Fujimori, K. Haneda, M. Mizuno, T. Inazu and H. Kumagai, Carbohydr. Res., 1998, 305, 415-422. e) M. Mizuno, K. Haneda, R. Iguchi, I. Muramoto, T. Kawakami, S. Aimoto, K. Yamamoto and T. Inazu, J. Am. Chem. Soc. 1999, 121, 284-290. 